This item was submitted to Loughborough's Research Repository by the author.

Items in Figshare are protected by copyright, with all rights reserved, unless otherwise indicated.

\title{
Material and efficient cause interpretations of the formative model: resolving misunderstandings and clarifying conceptual language
}

\section{PLEASE CITE THE PUBLISHED VERSION}

http://dx.doi.org/10.1007/s13162-013-0058-5

\section{PUBLISHER}

(c) Springer/@ Academy of Marketing Science

\section{VERSION}

AM (Accepted Manuscript)

\section{PUBLISHER STATEMENT}

This work is made available according to the conditions of the Creative Commons Attribution-NonCommercialNoDerivatives 4.0 International (CC BY-NC-ND 4.0) licence. Full details of this licence are available at: https://creativecommons.org/licenses/by-nc-nd/4.0/

\section{LICENCE}

CC BY-NC-ND 4.0

\section{REPOSITORY RECORD}

Cadogan, John W., Nick Lee, and Laura Chamberlain. 2019. "Material and Efficient Cause Interpretations of the Formative Model: Resolving Misunderstandings and Clarifying Conceptual Language". figshare. https://hdl.handle.net/2134/17718. 


\title{
MATERIAL AND EFFICIENT CAUSE INTERPRETATIONS OF THE FORMATIVE MODEL: RESOLVING MISUNDERSTANDINGS AND CLARIFYING CONCEPTUAL LANGUAGE
}

\author{
Nick Lee \\ Professor of Marketing and Organizational Research \\ Aston University \\ Aston Business School \\ Birmingham, B4 7ET, UK \\ Phone: $+44(0) 121-204-3152$ \\ Fax: +44(0)121-204-4917 \\ Email: n.j.lee@aston.ac.uk \\ John W. Cadogan \\ Professor of Marketing \\ Loughborough University and Lappeenranta University of Technology \\ School of Business and Economics \\ Loughborough, LE11 3TU, UK \\ Phone: +44-(0)1509-228846 \\ Fax: $+44(0) 1509-223960$ \\ Email: j.w.cadogan@lboro.ac.uk \\ Laura Chamberlain \\ Lecturer in Marketing \\ Aston University \\ Aston Business School \\ Birmingham, B4 7ET, UK \\ Phone: +44(0)121-204-3155 \\ Fax: $+44(0) 121-204-4917$ \\ Email: I.m.chamberlain1@aston.ac.uk
}

Accepted for publication in AMS Review

(Scheduled for publication - volume 4, issue 1, 2014) 


\begin{abstract}
This paper presents a causal explanation of formative variables that unpacks and clarifies the generally accepted idea that formative indicators are 'causes' of the focal formative variable. In doing this, we explore the recent paper by Diamantopoulos and Temme (2013), and show that the latter misunderstand the stance of Lee, Cadogan and Chamberlain (2013; see also Cadogan, Lee and Chamberlain 2013). By drawing on the multiple ways that one can interpret the idea of causality within the MIMIC model, we then demonstrate how the continued defence of the MIMIC model as a tool to validate formative indicators, and to identify formative variables in structural models, is misguided. We also present unambiguous recommendations on how formative variables can be modelled in lieu of the formative MIMIC model.
\end{abstract}

Keywords: Formative Variables; Measurement; Composites; Indicators; Theory; Causality; Ontology; Philosophy 
A recent paper by Diamantopoulos and Temme (2013) defends the continued use of the formative MIMIC model; that is, the use of a MIMIC model to validate formative indicators and identify formative latent variables in structural models. Diamantopoulos and Temme (2013) argue that the criticisms of the formative MIMIC model made in the work of Lee, Cadogan, and Chamberlain (2013), and Cadogan, Lee and Chamberlain (2013), are invalid. The latter two papers are not the first to cast doubt on the use of the formative MIMIC model, or on the formative model in general. Indeed, Diamantopoulos and Temme (2013, p. 160) also question such “eminent researchers as Roy Howell, John Edwards [sic we assume they mean Jeffrey Edwards], and Denny Borsboom - all known to be somewhat allergic to formative measures in general and their incorporation in MIMIC models in particular (e.g., see Borsboom et al. 2003; Edwards 2010; Howell 2013; Howell et al. 2007)”.

The present paper demonstrates that Diamantopoulos and Temme's (2013) arguments are fatally flawed, being as they are based on erroneous interpretations of our previously-cited work. The models that Diamantopoulos and Temme (2013) present as representations of our ideas are not representations of our ideas, and indeed are models that are conceptually flawed in light of our prior work ${ }^{1}$. As such, Diamantopoulos and Temme's (2013) commentary, which they intend as a confirmation of the utility and veracity of the formative MIMIC model, is misdirected. It seems that Diamantopoulos and Temme (2013), and others who maintain the use of formative MIMIC models, make key conceptual mistakes, most especially regarding fundamental principles of causality. To help future researchers who are contemplating whether, and how, to use formative variables, we present an explanation of the causality issues that are essential to making sense of formative modeling, and that are unappreciated in the relevant literature until now. In doing so, we advance a coherent conceptual language for discussing the causality issues bound up within formative models, and that clarifies the numerous misunderstandings and contradictions in existing literature. By reflecting on the causality issues inherent in the formative model, we

\footnotetext{
${ }^{1}$ Note, Model 6 in Diamantopoulos and Temme (2013) is clearly cited as Bollen and Bauldry’s (2011) idea in Lee, Cadogan, and Chamberlain (2013), and is not presented as “our” idea. In the latter paper, we use Model 6 as a speculative example of how other researchers might attempt to deal with the fundamental issues we point out, just as we use the logarithmic and multiattribute utility function examples (again, neither of which is our idea). While Diamantopoulos and Temme (2013) might accurately reproduce that model (although see the caveats pointed out later), it is not relevant to the present paper. As such, it is not part of this discussion. That said, we wonder why, while discussing Bollen and Bauldry's (2011) suggested model, Diamantopoulos and Temme (2013) do not also operationalize the other potential examples in the same section in Lee, Cadogan, and Chamberlain (2013).
} 
provide readers with the tools to see for themselves why it is logically impossible for the MIMIC to also be a 'formative measurement model'. We conclude by providing an unambiguous explanation of how formative variables should be implemented, demonstrating that beyond being an invalid mechanism for modeling formative variables, the formative MIMIC model is also an unnecessary complication.

\section{PROBLEMS WITH DIAMANTOPOULOS AND TEMME (2013)}

Diamantopoulos and Temme's (2013) intention is to debunk the work of Lee, Cadogan and Chamberlain (2013; see also Cadogan, Lee and Chamberlain, 2013). Unfortunately, they are unsuccessful because they misunderstand our stance, and the recommendations we derive from that stance. Specifically, Diamantopoulos and Temme (2013) construct five models in which the following features are common ${ }^{2}$.

(1) A focal latent variable, $\eta 1$ (satisfaction), is related to a set of reflective items.

(2) The same focal latent variable, $\eta 1$ (satisfaction), is also related to one or more formative items.

Some initial reflections can be made on the Diamantopoulos and Temme (2013) models before we begin our more substantive rebuttal to their comments on our own work. First, Diamantopoulos and Temme (2013) do not define their central construct of "students' satisfaction with the university cafeteria” (ibid, p. 160), beyond giving it that name. Second, they do not show the reader the content or wording of their formative and reflective items, only referring to them as $x$ s and $y$ s. Both these problems directly contradict our explicit advice that "the conceptualization process should be far more rigorous than simply giving a theoretical idea a name" and that "the definition of a formative variable must include the components [i.e. items] and the way they are combined together" (Cadogan, Lee, and Chamberlain, 2013, p. 47-48). In light of this, it is hard to see why Diamantopoulos and Temme (2013) consider their work to

\footnotetext{
${ }^{2}$ Unfortunately, Diamantopoulos and Temme’s (2013) Model 6 is not clearly specified (e.g., we do not know how the composites in are created). Thus, aside from the issues we highlight in the previous footnote (which mean we are uninterested in the Model 6), we would be unable to discuss Model 6 even if we were to be interested in it.
} 
offer any insight into whether our stance on formative modelling is defensible. Of course, the fact that the reader never gets to see empirical results other than the general fit statistics, and selected results that Diamantopoulos and Temme (2013) choose to report, is also problematic.

Beyond these issues, there are more fundamental reasons why Diamantopoulos and Temme's (2013) critique of our work, and their use of five models to do so, are invalid. To explain our reasoning, it is important that we clarify our assumptions regarding what the Diamantopoulos and Temme’s (2013) models imply in terms of measurement. Unfortunately, Diamantopoulos and Temme (2013) are not clear about which of the items in their models they consider to be measures of the focal construct ( $\eta 1$ ), and which (if any) are not - however, we think we can assume that their formative (i.e., all their exogenous) items are viewed as measures of $\eta 1$, since Diamantopoulos and Temme (2013, e.g. p. 160) repeatedly refer to "formative measurement" (particularly in relation to the avowed intention of the antagonists of their story; the hypothetical satisfaction researchers). However, what of the reflective items? Although no stance towards these items is specified by Diamantopoulos and Temme (2013), according to Diamantopoulos and Winklhofer (2001, p. 272), the reflective items represent "the essence of the construct the index purports to measure” and, according to Diamantopoulos (2013, p.33), given a set of exogenous observed formative indicators (the $x$ s) and a set of endogenous observed reflective indicators (the $y$ ), one can view "both the $x$ s and the $y s$ as content-valid indicators of $\eta$, with the $x$ s being formative and the ys being reflective” (Diamantopoulos 2013, p. 33). Further, Diamantopoulos (2013) offers a strident criticism of Cadogan, Lee, and Chamberlain (2013) for using reflective items in a MIMIC model that do not represent content valid indicators of a construct. As a result, assuming that they are consistent with the prior work of Diamantopoulos, we must infer that Diamantopoulos and Temme (2013) believe that their reflective items are also measures of the focal construct $(\eta 1)$. As such, and again following Diamantopoulos (2013), the reflective indicators are apparently measuring the same thing as the formative items. Accordingly, our reading of their work leads us to believe that, in general, Diamantopoulos and colleagues believe that the MIMIC model provides a way to simultaneously measure a construct using both formative and reflective items, and more specifically, that Diamantopoulos and Temme's (2013) satisfaction example contains what they believe to be content valid formative and reflective measure of the same construct (satisfaction). 
Given the above then, it is clear that none of Diamantopoulos and Temme's (2013) Models 1-5 represent our ideas of appropriate formative modeling. Rather, our stance is that when one is constructing formative variables, one should not include reflective items at all. If one must model a formative variable, then it must be modelled using the formative items alone, not with formative items plus some reflective items. It is a fact that including reflective items changes the meaning of the latent variable so that the model no longer contains a formed variable (Markus and Borsboom, 2013; Rigdon, 2013; Howell, 2013). As a result, including reflective items in the model renders the model invalid in the context of the original theory of a formative variable. Applying this reasoning to Diamantopoulos and Temme’s (2013) five models, then, we can conclude that none of them is valid. In essence:

(1) Diamantopoulos and Temme (2013) show that a collection of MIMIC-alike models perform quite poorly in terms of model fit in comparison to the "The original MIMIC model” (ibid, p. 161).

(2) However, the comparison is meaningless, since the original MIMIC model together with the MIMIC-alike comparison models are all invalid methods of modeling formative variables.

(3) As a result, the recommendations Diamantopoulos and Temme (2013, p. 167-8) present for modeling formative variables all rest on invalid assumptions. In other words, their recommendations are wrong, and should not be adhered to. We discuss this matter in more detail later in the paper, and provide unambiguous guidance on how formative variables must be modeled.

To summarize, Diamantopoulos and Temme (2013) try to show that a series of formative models containing reflective items - models that our work shows to be invalid - do not result in good fit. So, their intended criticism of our work is irrelevant because it incorrectly assumes that the MIMIC-alike models they propose are models that we advance (in part or in whole) as potentially valid alternatives to the formative MIMIC model. We simply do not do this - our past publications do not advance these models (in part or in whole). The fact that some of the models 
perform badly says nothing about any of our prior work, and neither can one reject our logic based on these results.

Of course, a central feature of Diamantopoulos and Temme's (2013) error is to incorrectly assume that the original formative MIMIC model is a valid formative variable model against which comparisons can be made. Indeed, we do not have to cite ourselves to show that there is a growing acceptance that the formative MIMIC model is a flawed tool (e.g., Borsboom et al. 2003; Borsboom, 2005; Edwards 2011; Howell 2013; Howell et al. 2007; Markus and Borsboom, 2013; Rigdon, 2013). A valid question, then, regards why some scholars remain wedded to the formative MIMIC model in the face of considerable evidence to the contrary much of which is even cited by Diamantopoulos and Temme themselves. We speculate that a reason for the formative MIMIC model's continued defense is somehow bound up with fundamental misunderstandings of the nature of measurement and causality. In the rest of this paper, we untangle the conceptual mistakes that scholars are making in continuing their support of the formative MIMIC model. In doing so, we provide a coherent conceptual language to enable scholars to more clearly progress theory development and engage more productively with the wider research community.

\section{CAUSAL INTERPRETATIONS USED IN THE FORMATIVE MODEL}

Essential to Diamantopoulos and Temme's (2013) formative MIMIC model is the idea of causality. They themselves note that the formative indicators in a formative MIMIC model are "causal” (p. 160). Yet Diamantopoulos and Temme refrain from explaining what they mean by "causal”. Our prior work expends considerable effort in defining one type of cause (see Lee, Cadogan, and Chamberlain, 2013; Cadogan, Lee and Chamberlain, 2013), from a generally Humean perspective ${ }^{3}$. However, there are multiple ways of defining causation (see Aristotle's Metaphysics Book 5, Part 2). In this paper, we extend our prior discussions of causality, to suggest that two different meanings of cause are often implicitly applied to the formative model, and that the conflation of these two types of cause is a core reason for the continued

\footnotetext{
${ }^{3}$ By this we mean that it is necessary for there to be two separate events/entities for a cause-effect relation to exist. In other words, one thing cannot cause itself. This is fundamental to Hume's accounts of cause (see A Treatise of Human Nature and An Enquiry Concerning Human Understanding).
} 
misunderstanding of the ontology of the formative variable evident in work such as Diamantopoulos and Temme (2013), and the continued application of the formative MIMIC model in research.

\section{Material Causation}

For most advocates of the formative model, a key notion is that the formative focal variable is made up of, or is formed by, its formative indicators. Typically, the formative model is represented using a mathematical structure as shown in equation 1 (Diamantopoulos, Reifler and Roth 2008, p. 1205):

\section{Equation 1}

$$
\eta=\sum_{i=1}^{n} \gamma_{i} x_{i}+\zeta
$$

where $\eta$ is the formed focal variable, the $x i$ are the set of formative indicators that define $\eta$ (the $x$ s are the "stuff of" $\eta$ ), and the $\gamma \mathrm{i}$ are parameters that represent a causal relationship between the $x i$ and $\eta$. Typically, advocates of the formative model also include a $\zeta$ term, which they define as being a portion of $\eta$ that is not contained in the set of $x$ s (e.g. Diamantopoulos and Winklhofer, 2001). As such, the formative focal variable is one that "receives its meaning from the [indicators, where]... each indicator captures a specific aspect of the construct's domain [and where]... omitting an indicator potentially alters the nature of the construct" (Diamantopoulos, Reifler and Roth 2008, p. 1205), since each indicator is "part of the construct itself" (Diamantopoulos and Winklhofer, 2001, p. 271). Within the context of this definition of the formative model, it is common for the formative indicators (i.e., the $x$ variables that make up the substance the formative focal variable) to also be described as cause or causal indicators (as we observe in Diamantopoulos and Temme's work). 
Fundamental to the formative model as defined above is the notion of material causation, where a cause is that of which the final consequence is made (see Aristotle's Physics Book 2, part 3) ${ }^{4}$. A material causality interpretation of the formative model defines cause in terms of the material composition of the formative focal variable: the formative indicators are cause indicators because they are the material of the construct. Thus, a formative variable is a "combination[...] of variables” (Fornell and Bookstein 1982, p. 292), "a linear sum of a set of measurements" (Bagozzi, 1994, p. 332), or "a weighted sum of the item scores” (Markus and Borsboom, 2013, p. 82).

Within this material causality perspective, the formative variable cannot be said to describe an attribute or property that exists independent of the formative indicators. While we formally introduce the specific 'material cause' terminology to formative modeling theory in this paper, it is clear that in our prior work (e.g., Lee, Cadogan, and Chamberlain, 2013; Cadogan, Lee and Chamberlain, 2013) we argue that such an interpretation of the formative model is the only one that should be called 'formative'. A key implication of the material causation interpretation of the formative model is that one cannot separate the formative focal variable from the constituent variables that form it, and so one cannot claim that (a) the formative focal variable is made of different conceptual 'stuff' from its material constituent variables, or that (b) the formative focal variable is somehow a variable that is separate from the constituent variables.

Importantly, a mistake made by many authors is to suggest that simply adding an error term to a latent variable model can by itself change the formative model from this material interpretation, and imbue the formative latent variable with some meaning or existence independent of the indicators that form it (e.g., Diamantopoulos and Winklhofer, 2001). In fact, if one wishes to maintain the material interpretation of the formative model - where the indicators are intrinsic parts (i.e., are the material) of the construct definition as specified by the various authors above the error term is not easily interpreted; it does not appear to have an obvious meaning. Clearly, if one were take away the material stuff that makes up the formed variable, there would be nothing

\footnotetext{
${ }^{4}$ In a similar way, Markus and Borsboom (2013) suggest that the formative model could represent formal causation, which Aristotle defines as describing the form of the final result (i.e., the shape, or appearance of the thing). However, this type of cause does not seem to represent the definitions of formative variables quoted herein as accurately as the material cause interpretation does.
} 
left (there would be no residual error), so what could error be? We suggest that an error term should play no role in the formative definition of a construct, and Diamantopoulos and Temme (2013) appear to agree with us, arguing that making "the error term, $\zeta 1$, part of the definition of the formative latent variable... is completely nonsensical” (p. 165). Yet at the same time, Diamantopoulos and other formative modeling advocates argue that equation 1 defines the formative variable, and equation 1 contains an error component. Borrowing Diamantopoulos and Temme's terminology, we can only conclude that equation 1 contains a nonsensical component (an error term) that should not be there. This problem is a conceptual issue, not an empirical modeling one, and the only solution, from a material causation perspective, is to drop the error term from equation 1.

Take the example of Socioeconomic Status (SES) that we discussed at length in Cadogan, Lee, and Chamberlain (2013). If SES is defined as the combination of income, occupation, and education, and "the indicators determine the latent variable...[and] its meaning" (Diamantopoulos, Riefler, and Roth, 2008, p. 1205) as in the material cause interpretation, then there is simply no logical way to justify SES as being anything more than the indicators. SES defined this way is simply a summary of the indicators, not an attribute that somehow exists apart from them. This is what material causation means, and placing an error term on this definition of SES does not change this basic fact.

\section{Efficient Causation}

There is an alternative interpretation of causality that is fundamentally different to material causation, and that is efficient causation (again, see Aristotle's Physics Book 2, part 3). Efficient causation is the type of causality that is closest to the Humean view articulated in our earlier work, as well as the view that is generally understood when laypeople talk about cause, and it refers to the role that one entity plays in producing a change in a separate entity. Efficient causation is the sort that requires two (or more) separate entities - a cause and one (or more) effects (entities that are changed as a result of a change in the cause). Examples of this causal interpretation of formative variables can also be found in existing literature, such as when Bollen and Davis (2009) use Blalock’s (e.g. 1964; 1971) idea that race and gender could be considered 
"causal indicators" of exposure to discrimination. Another even clearer example is found in Blalock (1971, p. 29-30), where a subject's frustration can be "caused” by hunger and fatigue.

While none of the above-cited authors attempt to address the nature of causation that they are invoking, it is at least obvious that in such cases, the indicators are not "part of the construct itself" (Diamantopoulos and Winklhofer, 2001, p. 271) and it simply cannot be the case that "the indicators determine the latent variable...[and] its meaning” (Diamantopoulos, Riefler, and Roth, 2008, p. 1205). In fact, the attributes in question (exposure to discrimination, fatigue) are not formed as "explanatory combinations of indicators" (Fornell and Bookstein 1982, p. 292), but are instead existent properties of the subject, quite separate conceptually and materially from the indicators (e.g., race, hunger). So, here the material cause interpretation cannot possibly apply.

Dimantopoulos and Temme (2013, footnote 14) provide a similar example, where they suggest one could gauge a latent variable of 'alcoholization' (a variable they do not define, unfortunately) by using the number of glasses of different alcohol types consumed as a set of "formative indicators" (ibid). They also suggest that the same set of indicators could be used to construct what they call an observed variable of 'total amount consumed' of alcohol. The 'total amount consumed' variable is clearly defined in a material causation way. Alcoholization, on the other hand, appears to be a property of the test subject that is separate to the amounts of alcohol consumed: the latter (the actual alcoholic drinks consumed) seem to be efficient causes of alcoholization (in other words, alcohol consumption activity is antecedent to, but not the same conceptual entity as, alcoholization) ${ }^{5}$.

A significant problem is that the formative model literature often conflates efficient and material causation, implicitly using efficient causation as a core mechanism on top of material causation. For example, in the context of a formative (or cause) indicator ( $x 1$ ), and a formative focal variable $(\eta)$, formative model advocates (and especially formative MIMIC model advocates)

\footnotetext{
${ }^{5}$ If it was the same entity, then 'alcoholization' would just be another name for 'total amount consumed'. That said, we certainly agree with the principle that the same indicators could be used in different model definitions. Indeed we suggested exactly that in Lee, Cadogan, and Chamberlain (2013). Interestingly, we were criticised by

Diamantopoulos (2013, p. 35 original emphasis), for using "these same indicators as items defining a composite of a different construct". Diamantopoulos (ibid) later says "I leave it to the reader to decide for himself/herself the utility of this exercise". However, it seems that Diamantopoulos has come around to the idea, since he co-opted it in Diamantopoulos and Temme (2013).
} 
often assume efficient causation whereby the $x 1$ and the $\eta$ variables are separate entities, so that a change in the $x 1$ entity brings about some change in the $\eta$ entity. Based on efficient causation, equation 1's specification of the formative variable is a simple regression, in which "the [formative] latent variable $[\eta]$ is a separate entity from its indicators [the $x \mathrm{~s}$ ]" (Diamantopoulos 2013, p.32). It is important to emphasize the assumption Diamantopoulos is making here - he is proposing that the formative indicators are not the same things as the formative focal variable: he is saying that the focal formative variable is endogenous to those formative indicators, such that "the latent variable $[\eta]$ is the dependent variable and the indicators [the $x \mathrm{~s}$ ] are the explanatory variables” (Diamantopoulos, Reifler and Roth 2008, p. 1205).

Unfortunately, there is a logical inconsistency that emerges from simultaneously imbuing the formative model with both material and efficient causal interpretations. Specifically:

- Material causation means that the formative focal variable is the same material as its constituent indicator $(x)$ variables, and is not a separate conceptual entity from its constituent $(x)$ variables: the formative variable is constructed using the constituent variables - it is the constituent variables. However:

- Efficient causation means that the formative focal variable is not the same material as the $x$ variables - the formative focal variable is a separate entity from the $x$ variables (Diamantopoulos 2013), and is impacted on by those $x$ variables.

One can see that it is logically impossible for a formative model to contain both material causation and efficient causation: only one kind of causation can be at work in a formative model. Material causation makes good sense in the formative model - it is implicitly how most people view formed things. Yet if a formative model operates on a material causation basis, then it cannot also operate on an efficient causation basis: that is, if the formative focal variable is the same material as its constituent indicator $(x)$ variables, then the constituent indicator variables cannot also operate as efficient causes of the formed variable - because there is no separation between the indicators and the formed variable, they are the same thing, as we state clearly in Lee, Cadogan, and Chamberlain (2013). Efficient causation requires the cause and the effect to 
be separate entities. As a result, under the material causation interpretation of the formative model, the formative MIMIC model makes no sense, since the latter is grounded in the process of estimating the magnitude of the cause-effect relationships (i.e., efficient causes) between some formative indicator variables ( $x \mathrm{~s})$ and an endogenous focal variable.

If a formative model operates on an efficient causation basis, then the model cannot also operate on a material causation basis. That is, if the formative indicators impact on the formative focal variable in a cause-effect type way (efficient causation), then the formative focal variable cannot also be the same material as its formative indicators. One entity cannot be an efficient cause of itself. So, to retain the formative MIMIC model, one must reject the material causation interpretation of the formative model. Yet, interpreting the formative model in terms of efficient causation leaves one with a rather odd formative model - one in which the variables that supposedly 'form' the formative focal construct are actually different entities from (i.e., are antecedent efficient causes of) the focal variable. Here, it becomes impossible to tell the difference between a formative variable model (where a dependent variable is predicted using some independent variables) and any other structural model (in which a dependent variable is predicted by some independent variables), as also pointed out by Markus and Borsboom (2013). In essence then, all structural models that have cause-effect relationships would become formative models under the efficient causation interpretation of the formative model! ${ }^{6}$ A further problem with the efficient causation interpretation of the formative model is that there is no place in it for 'true' formative variables - those that are constructed as simple composites of variables, where the formative focal variable is materially comprised of the formative indicators.

\footnotetext{
${ }^{6}$ Diamantopoulos (2013, p. 33) cites himself in attempting to overcome this conceptual impossibility by saying that "how a particular MIMIC model should be interpreted 'depends on the conceptual interpretation attacked [sic] to the relationships between $\eta$, the xs and the ys' (Diamantopoulos 2011, p. 346, original emphasis)". As should now be apparent, this line of thinking only makes sense if one is erroneously attempting to simultaneously invoke material and efficient causal interpretations onto the MIMIC model. Interestingly, Diamantopoulos (2013) criticizes Lee, Cadogan and Chamberlain, (2013) for advocating that formative models are not subject to empirical testing using the MIMIC model. Key pillars of Diamantopoulos' (2013, p. 35) criticisms of our arguments against the formative MIMIC are that it is wrong to set up a situation where "your auxiliary theory cannot be refuted (because it cannot be tested)" and that it is problematic that by rejecting the formative MIMIC model, we "provide no opportunity for assessing whether" the combination rules that we decide on for the creation of the formative variable "are indeed reasonable”. Diamantopoulos' stance on this front, then, appears to be at odds with his argument that it is up to a researcher's conceptual interpretation as to whether a MIMIC model contains a formative variable. We clearly show in the above that the conceptualization of a MIMIC model as a formative variable model is flawed, since it amounts to the same thing as a basic efficient cause model of antecedents - whatever terminology one places on it.
} 
Based on this reasoning, we can see that the only reasonable option is to reject efficient causation as a viable interpretation of the formative model, and to rely exclusively on material causation as a foundational interpretation platform for formative models. Efficient causation should be considered for structural models only, in which the cause-effect entities are separate, and where the cause-effect entities are not also being considered to simultaneously contain material causality. Of course, if we reject efficient cause as a mechanism underpinning the formative variable, then we must also reject another element of equation 1, the $\gamma$ terms. Specifically, the $\gamma \mathrm{i}$ represent the efficient causal relationships between one set of entities (the $x$ ) and a separate entity ( $\eta$ ). Accordingly, if one accepts the material cause interpretation of a formative variable, one must reject the efficient cause interpretation, and as a result, one must also reject the idea that equation 1 provides a representation of a formative variable. Specifically, one must reject the ideas promoted by equation 1 that says that (a) there is somehow some error in the definition of the formative variable (since this is nonsense, according to Diamantopoulos and Temme), and that (b) there are (efficient) causal relationships between the formative indicators ( $x$ s) and the formative focal variable $(\eta)$, and that the magnitude of these causal relationships can somehow be estimated from some data.

At the risk of making unwarranted assumptions, we now turn briefly by way of example to the task that Diamantopoulos and Temme’s (2013, p. 160) hypothetical researchers set themselves to "estimate the parameters of a formative specification of their students' satisfaction with the university cafeteria”. In doing so, the original intention is to estimate these parameters by way of a MIMIC model. Unfortunately, the success or otherwise of this endeavor is impossible to determine from Diamantopoulos and Temme's report of their activities. Specifically, without a definition of the focal construct, and the items used, it is impossible to see whether or not the researchers are using a material or efficient cause interpretation. In fact, if one was to conceptualize 'satisfaction' as an attribute of each student ${ }^{7}$, one could conceptualize the so-called formative items as antecedent causes of variation in satisfaction ${ }^{8}$, and the reflective items as measures. This is the efficient cause definition, and it is one that we argue is consistent with a

\footnotetext{
${ }_{8}^{7}$ Probably the most logical definition.

8 That is, one could decide not to interpret the items as entities that form, in a material sense, a focal variable.
} 
MIMIC model approach, as do others (e.g. Markus and Borsboom, 2013). However, it is not a formative model, as the items would not be definitionally essential to the construct, and so would not operate as material causes. There is absolutely no difference between the efficient cause definition and a structural model of antecedents, and there is no conceptual advantage to be gained by pretending that calling it a 'formative measurement model' imbues it with some new properties, such as being measurement. If the formative items truly do define the material of the construct itself, then a material cause model is in operation. This model does describe the formative approach, but equally it is then impossible to use the MIMIC model to operationalize it. Below, we give unambiguous advice on how best to model formative variables (i.e., those that adhere to a material cause interpretation).

\section{HOW SHOULD FORMATIVE VARIABLES BE MODELED?}

The previous discussion on the causal underpinnings of the formative model should clarify that formative MIMIC models cannot exist, since they require that researchers simultaneously invoke two different kinds of causality interpretation on the formative model. On the one hand, a formative model imposes a material causality interpretation, in which the formative indicators are the material substance of the formed focal variable. At the same time, the MIMIC model itself imposes an efficient causality interpretation, in which the formative indicators are not the material substance of the formed focal variable, but rather are different entities to the formed focal construct. The MIMIC model is fine in itself, but it is not the mythical 'formative measurement model': the MIMIC model is simply a model of the antecedents to a reflectively measured attribute (Markus and Borsboom, 2013).

This inherent contradiction in the ontology of the formative variable can be resolved in two ways. The first way (the most sensible approach) is to remove the efficient causality interpretation from the formative model (i.e., equation 1 would no longer be used as a representation of the formative variable). Rather, a formative variable would simply be a set of variables that are combined into some useful composite, with no efficient cause assumption imposed on the formative variables in respect to the formed focal construct. The second wayis to remove material causality from the formative model: rather one accepts that one has a an entity 
$(\eta)$ that is different from (not the same conceptual entity as) the group of $x$ indicators in equation1, and that the $x$ s have potential cause-effect type outcomes on $\eta$. Adding some reflective indicators would make this model a MIMIC model, but not a traditional model of a formative variable (since the xs would not be the stuff of $\eta$ ). It is only under the efficient causality interpretation of the MIMIC model that equation 1 makes sense. As such, equation 1 does not provide a logical representation of a formative variable ${ }^{9}$.

The result is that any model which a researcher believes is formative and that is implemented using a MIMIC model is not doing what the researcher thinks it is doing: it is not modeling a formative variable in which material causality is operating, for instance. If material causality is not operating then, possibly, efficient causality is operating, but a model in which efficient causality is operating and where material causation is not operating is not what anyone would recognize as a formative model in the definitional terms of prior work (e.g., Diamantopoulos and Winklhofer, 2001; Diamantopoulos, Riefler, and Roth, 2008). In fact, it is simply a model of some antecedent variables to a reflectively-measured attribute, as pointed out repeatedly by ourselves and others (e.g., Markus and Borsboom, 2013). Seen in such a light, the commonlycited problems of the MIMIC model, such as interpretational confounding (e.g., Howell, Brievik, and Wilcox, 2007; Burt, 1976) and referential instability (Markus and Borsboom, 2013) are simply natural features of a structural model of a MIMIC form. They are, in fact, essential properties of a model which tests the impact of the observed antecedents on the reflectivelymeasured consequence. They only become problematical when one erroneously interprets the MIMIC model as a validation tool for formative indicators, or a way of identifying a formative latent variable in a structural model.

\section{Modeling formative variables in structural models}

So far, this paper shows that Diamantopoulos and Temme’s (2013) efforts to present (and subsequently criticize) Lee, Cadogan and Chamberlain (2013) and Cadogan, Lee and

\footnotetext{
${ }^{9}$ Possibly, equation 1 is the origin of all the confusion in the minds of formative MIMIC model advocates. If one assumes that material causation is what a formative variable is, but then superimpose equation 1 onto that definition, as Diamantopoulos and Winklhofer (2001) do (themselves borrowing from Bollen [e.g. Bollen and Lennox 1991] and collegues), then one will end up with the problems outlined here, and a belief that the MIMIC model can be used to model formative variables. We advocate eliminating equation 1 from the formative variable definition.
} 
Chamberlain's (2013) ideas regarding how a formative focal variable should be modeled miss the mark because the five models they produce all contain MIMIC-alike elements, and specifically, all contain reflective items. Our unambiguous position, presented clearly in Lee, Cadogan and Chamberlain (2013) and Cadogan, Lee and Chamberlain's (2013), is that reflective items have no place in the construction of a formative variable.

The current paper also shows that a material causality interpretation of the formative model is the most promising, and that an efficient causality interpretation of the formative model is not appealing since, for instance, it excludes from consideration models that would traditionally be defined as formative.

How then, should a formative variable be modeled in a substantive theory test (especially since we know that researchers are unlikely to measure their formative indicators without error)? If one must model variables as formative in substantive theory, as we now show, our view is that one must somehow look to model the error in a way that avoids the MIMIC model problem, and one must also explicitly specify a priori how much error is in that formative composite, by using some conceptual argument. Combined with the specification of weightings for the actual formative indicators - and the avoidance of using reflective indicators in a MIMIC model - one might create a useful proxy composite. This approach is superior to estimating meaningless loadings (in the context of a formative variable) using a MIMIC model.

With this in mind, we present some clear recommendations for modelling formative variables. Of course, we assume that the researcher has good reason to model a formative variable in the first place. Our approach is simple, and rests on guidelines in Lee, Cadogan and Chamberlain (2013) and Cadogan, Lee and Chamberlain (2013).

(1) Define the formative variable, explicitly identifying the variables that are to be combined and the way they are to be combined together (i.e., the weights, and any mathematical combination rules).

(2) Create an observed score for the formative variable using data on the defining variables and 
the combination rules that are specified in the variable’s definition.

(3) Use the latter observed score to undertake some kind of modeling job. For instance, calculate an average value for the formative variable across a sample; compare the average score for the formative variable across different samples, or over time.

Just to make things clear, nowhere do we advocate estimating a model using reflective items and formative items.

Of course, the approach above creates an observed composite score, and does not explicitly incorporate recognition that the formed composite was created from imperfectly measured 'indicators'. However, it is also possible to model a formative variable as a latent variable, provided that one goes about it the right way. We use advertising expenditure as an example. Specifically, when one creates a formative composite score C (observed advertising expenditure), one is combining the observed scores obtained from a set of formative indicators. In Figure 1, for instance, observed advertising expenditure $C$ is obtained by simply summing observed TV expenditure (x1), observed radio expenditure (x2) and observed newspaper expenditure (x3).

\section{Figure 1 about here}

However, x1, x2 and x3 are merely the observed scores obtained for the real quantities of $\xi 1, \xi 2$, and $\xi 3$ respectively, such that $\mathrm{x} 1, \mathrm{x} 2$ and $\mathrm{x} 3$ contain measurement error (e1, e2, and e3 respectively). As a result, and as Figure 2 shows, the observed composite score, C obtained by combining $\mathrm{x} 1$, $\mathrm{x} 2$ and $\mathrm{x} 3$ will differ from the true composite score value, $\mathrm{c}$, that one would obtain if one could combine $\xi 1, \xi 2$, and $\xi 3$. The difference between $\mathrm{c}$ and $\mathrm{C}$ is e4, where e 4 represents some combination of the measurement errors e1, e2 and e3. Here, we place c and the error term e4 in hexagons rather than ellipses, so as to differentiate c and e4 from being real entities (i.e., things which exist independent of their indicators: see Cadogan, Lee, and Chamberlain, 2013).

Figure 2 about here 
If researchers want to conduct modeling with c, C, and e4 in a structural equation modeling package, then, they must make some assumptions about the magnitude of e1, e2 and e3 (i.e., make assumptions regarding the reliability of the $\mathrm{x} 1, \mathrm{x} 2$ and $\mathrm{x} 3$ measures), and use the information to calculate the error variance of e4. For instance, if $\mathrm{x} 1$ is reliable at $. \mathrm{r} 1=70$, $\mathrm{x} 2$ is reliable at $\mathrm{r} 2=.74$ and $\mathrm{x} 3$ is reliable at $\mathrm{r} 3=.81$, then the average reliability of the $\mathrm{xs}$ is .75 . One might decide, therefore, that an improvement over assuming that $\mathrm{C}$ provides an errorless assessment of $\mathrm{c}$ is to assume that $\mathrm{C}$ has a reliability $\mathrm{r} 4=.75$ as a barometer of $\mathrm{c}$. Accordingly, borrowing from Jöreskog and Sörbom (1993), c could be modeled in a structural model as a latent variable with an observed score of $\mathrm{C}$ (loading set at 1 to set the metric), and with an error in assessment of $\mathrm{C}$ set at $(1-\mathrm{r} 4) * \operatorname{VAR}(\mathrm{C})$ (where $\operatorname{VAR}(\mathrm{C})$ is the variance of the observed score for C).

Note that the observed C score is not assumed to be caused by a single entity, c, since c is itself a multidimensional composite. Rather:

Equation 2: $\mathrm{x} 1=\gamma 1 * \xi 1+\mathrm{e} 1$

Equation 3: $\mathrm{x} 2=\gamma 2 * \xi 2+\mathrm{e} 2$

Equation 4: $\mathrm{x} 3=\gamma 3 * \xi 3+\mathrm{e} 3$

Equation 5: $\mathrm{c}=\xi 1+\xi 2+\xi 3$

Equation 6: e4 = e1 + e2 + e3

Equation 7: $\mathrm{C}=\mathrm{x} 1+\mathrm{x} 2+\mathrm{x} 3$

Where the $\gamma$ values represent the loading of the true scores on the observed scores. Further:

Substituting equations 2, 3 and 4 into equation 7, gives:

Equation 8: $\mathrm{C}=\gamma 1 * \xi 1+\gamma 2 * \xi 2+\gamma 3 * \xi 3+\mathrm{e} 1+\mathrm{e} 2+\mathrm{e} 3$

One can summarize equation 8 with: 
Equation 9: $\mathrm{C}=\mathrm{c} \gamma^{\prime}+\mathrm{e} 4$

Where $\gamma^{\prime}$ is a diagonal matrix containing $\gamma 1, \gamma 2$ and $\gamma 3$ loadings respectively on the diagonal (with off diagonal values of zero).

What to do with Diamantopoulos and Temme's guidelines for modeling formative variables

Diamantopoulos and Temme (2013) also present some recommendations regarding the use of formative models. Their recommendations are inherently based on the notion that the formative MIMIC model is valid, and that it provides information on a formative variable that is useful. Yet knowing that the formative MIMIC model is invalid, how do Diamantopoulos and Temme's (all quotes from 2013, p. 167-8) recommendations change?

Recommendation 1: "Ensure that the set of formative indicators used to operationalize the focal construct is consistent with the construct definition; all facets of the construct explicitly specified in the conceptual definition should be included as indicators". We wholeheartedly agree. If a construct is defined as being made up of some variables, then those variables should ideally be used when one is operationalizing the construct. Indeed, this is also part of our own advice in Cadogan, Lee and Chamberlain (2013).

Recommendation 2: "Ensure that any change in the conceptual definition of the construct that broadens/narrows the latter's domain of content is followed by appropriate additions/deletions to the set of formative indicators". This is basically a restatement of recommendation 1 , and of course, we agree.

Recommendation 3: "Ensure that the set of reflective items used in a MIMIC model is conceptually related to the formative indicators". In light of the discussion herein, it is clear that Dimantopoulos and Temme (2013) are misguided on this front, because they are assuming that 
the formative MIMIC can be used to model formative variables. It cannot, as we show above, and so MIMIC models should not be used to identify or otherwise validate formative variables.

Recommendation 4: "Do not set the error variance of the formative latent variable to zero in the MIMIC model.” This recommendation is meaningless since the MIMIC model should not be used to identify a formative variable. Also, to set the error to zero in a MIMIC is essentially to make the claim that the antecedents explain $100 \%$ of the variation in a reflectively-measured endogenous attribute (Markus and Borsboom, 2013). Beyond this, the MIMIC model does not test a formative model's validity in any case, as we clearly show above.

Recommendation 5: "If you so desire, by all means use predefined weights for the formative indicators ... However, be brave enough to also test your choice of weights against freely estimated weights based on the empirical data at hand.” Again, we disagree with this statement, because testing for the weights assumes that there are efficient cause-type relationships between the formative indicators and the formative variable, which implies that the efficient causality interpretation is operating. As we demonstrate above, efficient causality contradicts the most basic principle of formed variable, the material causality requirement (which assumes the formed variable is the same material as the things that form it). Accordingly, no matter how "brave” they are, a researcher should not and cannot meaningfully "test” for weights, but should use predefined weights to create a formative composite.

Recommendation 6: "Resist, at all costs, any temptation not to test your predefined weights... be guided by the view that "the relationships between the constructs and the measures should also be thought of as hypotheses that need to be evaluated in addition to the structural paths" (Petter et al. 2007, p. 624)”. This is just wrong in the present context, and our comments in relation to recommendation 5 are relevant here as well. Any estimates from a formative MIMIC model are uninterpretable as tests of validity for formative indicators. As Borsboom, Mellenbergh and Van 
Heerden (2003, p. 209) point out in the same context, "roughly speaking, one could say that the idea of estimation is meaningful only if there is something to be estimated”.

Recommendation 7: "Do not replace the individual indicators with a single composite score based on predefined weights unless you can first demonstrate that your choice of weights is appropriate/reasonable”. We agree in principle with the general recommendation, and again give the same advice in our own work. However, we disagree that evidence for the appropriateness of formative weights can be discovered through empirical estimation of a MIMIC model. Rather, either direct evidence from the subject of measurement (e.g. the clinimetric approach we alluded to in Lee, Cadogan and Chamberlain, 2013), or theoretical and conceptual discussion and agreement are needed. Without this, there can be problems. For example, imagine one were to weight four formative indicators of a formatively defined variable as having equal weights, except for the fourth indicator which is weighted at 1/15th the weighting of the other three indicators. If the research community disagree with this weighting profile, and subsequently agree to change it (say, to all four indictors having equal weight), then the composite score obtained using the original weighting profile will produce results that are invalid in the context of the subsequently agreed equal weighting profile. Therefore we recommend that weights be carefully predefined, and recommend methods on this front in our prior work.

Recommendation 8: “Accept that working with MIMIC models and formative measures requires a lot of work”. We agree that formative models complicate things. Formative variables are, by definition composite variables, and so do not have singular conceptual meaning. As a result, including formative variables in conceptual models is fraught with conceptual and empirical pitfalls. We do not agree that working with formative MIMIC models is something that requires lots of work: MIMIC models should not be used to identify or otherwise validate formative variables (see also our comment on recommendation 3).

\section{CONCLUSION}


In the present paper, we respond to Diamantopoulos and Temme's (2013) criticism of our prior work (see Lee, Cadogan, and Chamberlain, 2013; Cadogan, Lee, and Chamberlain, 2013). We show that their critique is based on a flawed understanding of our own work, which has led them to present a set of theoretical arguments and empirical results that do not represent a significant challenge to our own argument that the use of the MIMIC model to validate formative indicators, and to identify formative latent variables in structural models, is flawed and misleading. In fact, our work in this regard is but a small part of a growing body of scholarly thought which casts serious doubt on the formative model in general (e.g., Borsboom et al. 2003; Borsboom, 2005; Cadogan and Lee, 2012; Edwards 2011; Hardin and Marcoulides, 2011; Hardin et al. 2011; Howell 2013; Howell et al. 2007; Lee and Cadogan, 2012; Rigdon, 2013; Wilcox et al., 2008). In fact, even in the short period since the appearance of our two papers in AMS Review, further work which exhibits significant commonality with many of our arguments is emerging (Markus and Borsboom, 2013).

In our view, the implications of such work are devastating to the idea of the formative MIMIC model, and in light of this, this study presents speculations on the reasons why some authors remain committed to promoting the MIMIC model to validate formative indicators, and to identify formative latent variables in structural models. It seems likely that a failure to appreciate the subtleties of meaning inherent to the notion of causality may be at the heart of this. Much of our paper is dedicated to explicating the material and efficient definitions of causality, and explaining how they can be used to clarify the appropriate use of the formative model. In doing so, we hope we give the formative model an appropriate place in the conceptual toolkits of marketing researchers - much as similar models are useful in clinical and other fields. What is clear though, is that there is no place for the MIMIC model as a tool to validate formative variable models, or to identify formative latent variables in structural models. We therefore provide some unambiguous guidelines for researchers who do wish to operationalize formative variable models.

With all of this in mind, we finally turn our attention to whether or not the formative model is a measurement model. Diamantopoulos and Temme (2013, e.g., p. 160) certainly seem to think so, given their repeated references to "formative measurement" and "formative measure[s]" (see 
also e.g., Diamantopoulos and Winklhofer, 2001, and many others). We argue otherwise in both Lee, Cadogan and Chamberlain (2013), and Cadogan, Lee, and Chamberlain (2013), and we are hardly alone in this (see also Bosboom, 2005; Borsboom et al. 2003; Cadogan and Lee, 2012; Edwards, 2011; Howell et al., 2007; Hardin et al., 2011; Lee and Cadogan, 2012; Wilcox et al., 2008). In fact, Markus and Borsboom (2013, p. 119-120) talk of the "unfortunate marriage of the terms 'formative' and 'measurement.....the formative model is not a measurement model, precisely because the notion of measurement presupposes that the measured attribute plays a causal role in the generation of test scores. Thus, the distinction between formative modeling and formative measurement is important. The terminology of formative measurement suggests that formative modelling can serve as a replacement of reflective models in a measurement framework, and we agree with Edwards (2010) that this is not the case. Measurement is always reflective. However, formative modeling can nevertheless play an important role in test analysis”. We could not say it better ourselves.

We are grateful to Diamantopoulos and Temme for their commentary on our work (see also Diamantopoulos 2013). In light of their comments we are able to refine our understanding of critical issues, and hone our arguments about those issues. In doing so, we believe that with the introduction of material and efficient causation into the lexicon of formative modeling, we at last provide a coherent conceptual framework for theorizing about formative variables.

Diamantopoulos and Winklhofer (2001, p. 271) are correct when they define formative indicators as being "part of the construct itself”. However, the implications of this definition are often not followed through in the later conceptualization and modeling of formative variables. The use of the formative MIMIC model (and perhaps equation 1), are instantiations of the conflation of a) material causation with efficient causation, and in turn b) attributes that exist apart from their indicators, and variables that are simply combinations of their indicators. Such confusion leads to serious flaws in the conceptualization and operationalization of formative variable model, as can be seen in research published over the last two decades. However, as we suggest in Lee, Cadogan, and Chamberlain (2013) fields such as clinimetrics and health economics present us with methods of forming composites that look eminently applicable to many situations of relevance herein (see Fayers and Hand, 2002; Feeney, 2006; Ryan and Farrar, 2000; Torrance et al. 1996). We support the appropriate use of the formative model - indeed, we 
believe the framework we introduce in this paper is the only way it can remain a viable model. However, while the MIMIC model works as a structural model of antecedents (efficient causes) it is simply incorrect to continue using the MIMIC model as a way of validating formative indicators (material causes). 


\section{REFERENCES}

Aristotle (1984) The Complete Works: The Revised Oxford Translation, vol. 1, Barnes, J. (ed.). Princeton University Press: NJ

Aristotle (1984) The Complete Works: The Revised Oxford Translation, vol. 2, Barnes, J. (ed.). Princeton University Press: NJ

Bagozzi, R. P. (1994) Structural Equation Models in Marketing Research: Basic Principles, in Principles of Marketing Research, Bagozzi, R. P. (Ed). Blackwell: Cambridge, Mass.

Blalock, H. M. (1964) Causal Inferences in Nonexperimental Research. W.W. Norton: New York, NY.

Bollen, K.A. \& Bauldry, S. (2011). Three Cs in Measurement Models: Causal Indicators, Composite Indicators, and Covariates. Psychological Methods, 16 (3), 265-284.

Bollen, K.A. \& Davis, W.R. (2009) Causal Indicator models: Identification, Estimation, and Testing. Structural Equation Modeling, 16 (3), 498-522.

Bollen, K.A. \& Lennox, R. (1991). Conventional Wisdom in Measurement: A Structural Equations Perspective. Psychological Bulletin, 110 (2), 305-314.

Borsboom, D. (2005). Measuring the Mind: Conceptual Issues in Contemporary Psychometrics. Cambridge, UK: Cambridge University Press.

Borsboom, D., Mellenbergh, G.J. \& van Heerden, J. (2003). The Theoretical Status of Latent Variables. Psychological Review, 110 (2), 203-219.

Burt, R.S. (1976). Interpretational Confounding of Unobserved Variables in Structural Equation Models. Sociological Methods and Research, 5 (1), 3-52.

Cadogan, J.C., Lee, N. \& Chamberlain, L (2013). Formative Variables are Unreal Variables: Why the Formative MIMIC Model is Invalid. AMS Review, 3 (1), 38-49.

Cadogan, J.W. \& Lee, N.J. (2013). Improper Use of Endogenous Formative Variables. Journal of Business Research, 66 (2), 233-241. 
Diamantopoulos, A. \& Winklhofer, H.M. (2001). Index Construction with Formative Indicators: An Alternative to Scale Development. Journal of Marketing Research, 38, 269-277.

Diamantopoulos, A. (2011). Incorporating Formative Measures into Covariance-Based Structural Equation Models. MIS Quarterly, 35 (2), 335-358.

Diamantopoulos, A. (2013). MIMIC Models and Formative Measurement: Some Thoughts on Lee, Cadogan, and Chamberlain. AMS Review, 3 (1), 30-37.

Diamantopoulos, A. and Temme, D.T (2013). MIMIC Models, Formative Indicators and the Joys of Research. AMS Review, 3 (3), 160-171.

Diamantopoulos, A., Riefler, P. \& Roth, K.P. (2008). Formative Indicators: Introduction to the Special Issue. Journal of Business Research, 61 (12), 1203-1218.

Edwards, J.E. (2011). The Fallacy of Formative Measurement. Organizational Research Methods, 14 (2), 370-388.

Fayers, P.M. \& Hand, D.J. (2002). Causal Variables, Indicator Variables and Measurement Scales: An Example from Quality of Life. Journal of the Royal Statistical Society A, 165 (2), 233-261.

Feeney, D. (2006). The Multiattribute Utility Approach to Assessing Health-Related Quality of Life. In A.M. Jones (Ed.) The Elgar Companion to Health Economics, Cheltenham, UK: Edward Elgar Publishing.

Fornell, C. \& Bookstein, F.L. (1982). A Comparitive Analysis of Two Structural Equation Models: LISREL and PLS Applied to Market Data. In C. Fornell (Ed.). A Second Generation of Multivariate Analysis, New York, NY: Praeger.

Hardin, A.M, \& Marcoulides, G.A. (2011). A Commentary on the Use of Formative Measurement. Educational and Psychological Measurement, 71 (5), 753-764.

Hardin, A.M., Chang, J.C.-J., Fuller, M.A., \& Torkzadeh, G. (2011). Formative Measurement and Academic Research: In Search of Measurement Theory. Educational and Psychological Measurement, 71 (2), 281-305. 
Howell, R.D., Breivik, E. \& Wilcox, J.B. (2007a) Reconsidering Formative Measurement. Psychological Methods, 12(2), 205-218.

Hume, D. (1985) A Treatise of Human Nature, Penguin Classics: London, England.

Hume, D. (2008) An Enquiry Concerning Human Understanding, Oxford University Press: Oxford, England.

Jöreskog, K., and D. Sörbom (1993), LISREL 8: User's Reference Guide. Chicago: Scientific Software International.

Lee, N. \& Cadogan, J.W. (2013). Problems with Formative and Higher-Order Reflective Variables. Journal of Business Research, 66 (2), 242-247.

Lee, N., Cadogan, J.W. \& Chamberlain, L. (2013), The MIMIC Model and Formative Variables: Problems and Solutions. AMS Review, 3 (1), 3-17.

Markus, K.A.\& Borsboom, D. (2013). Frontiers of Test Validity Theory. Routledge: New York: NY.

Petter, S., Straub, D., \& Rai, A. (2007). Specifying formative constructs in information systems research. MIS Quarterly, 31(4), 623-656.

Rigdon, E. E., (2013), Lee, Cadogan, and Chamberlain: An Excellent Point . . . But What about that Iceberg? AMS Review, 3 (1), 24-29..

Ryan, M. \& Farrar, S. (2000). Using Conjoint Analysis to Elicit Preferences for Health Care. British Medical Journal, 320, 1530-1533.

Torrance, G.W., Feeny, D.H., Furlong, W.J., Barr, R.D., Zhang, Y. \& Wang, Q. (1996). A Multiattribute Utility Function for a Comprehensive Health Status Classification System: Health Utilities Mark 2. Medical Care, 34 (7), 702-722.

Wilcox, J.B., Howell, R.D. \& Breivik, E. (2008). Questions about Formative Measurement. Journal of Business Research, 61(12), 1219-1228. 
Figure 1: The construction of the formative indicator C (Observed Advertising Expenditure)
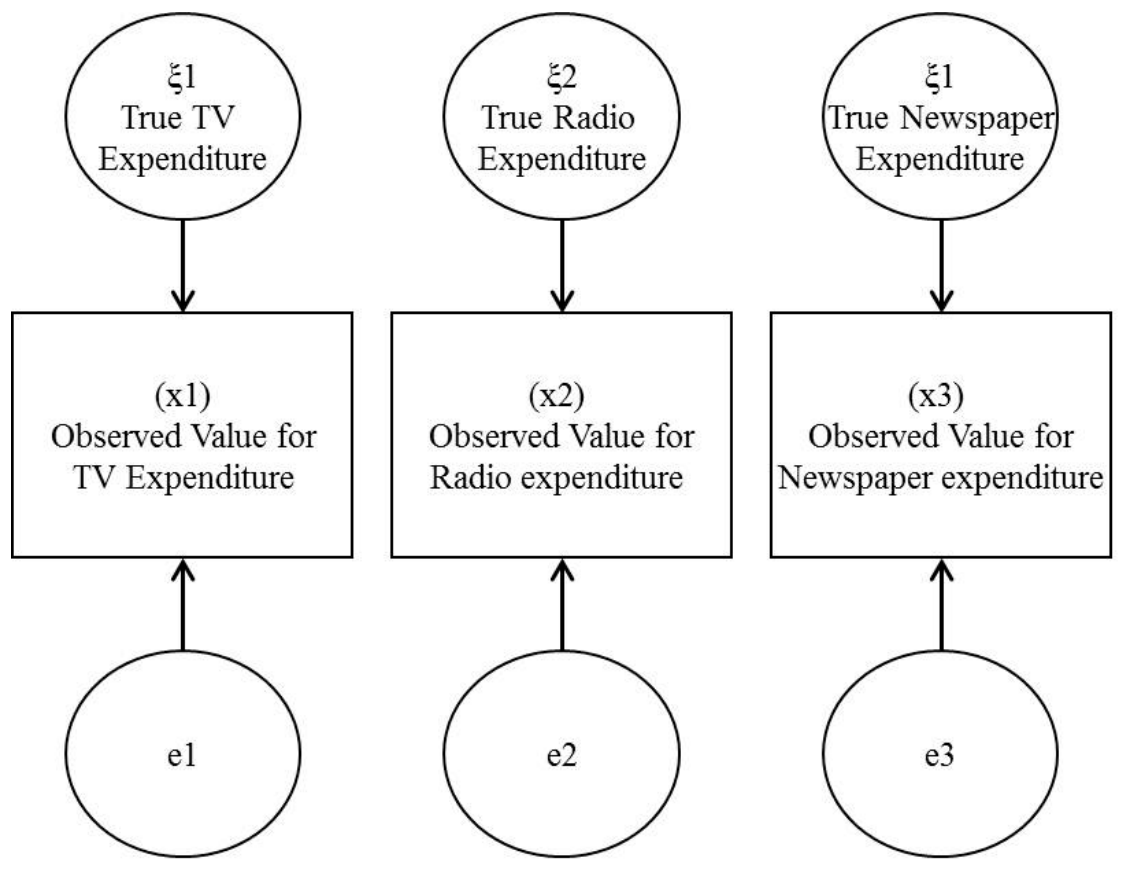

(C)

Observed Composite

Value for

Advertising

Expenditure

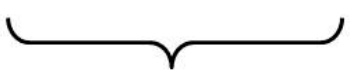

The observed composite score is obtained by summing $\mathrm{x} 1, \mathrm{x} 2$ and $\mathrm{x} 3$ 
Figure 2: Modeling the true composite value as an observed composite score with error

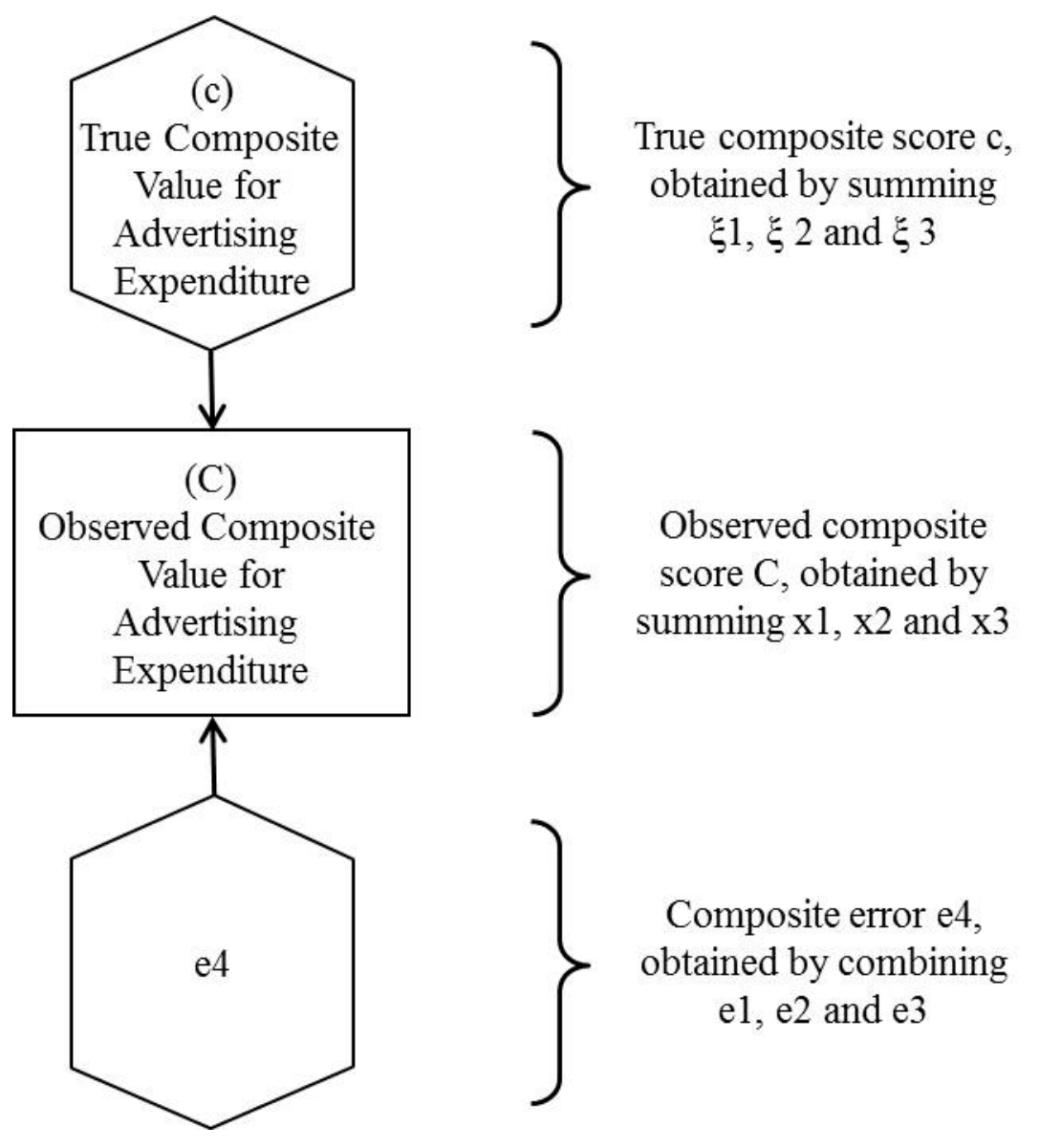

